\title{
Monoparesis Post Bronchial Artery Embolization: Clinicoradiological Discordance in Anterior Spinal Artery Infarct-Case Report and Review of Literature
}

\author{
Saurabh Gupta ${ }^{1}$ Siddharth Prakash ${ }^{1} \quad$ Ashima Mittal $^{1}$ \\ ${ }^{1}$ Department of Radiology, Dayanand Medical College and Hospital, \\ Ludhiana, Punjab, India
}

J Clin Interv Radiol ISVIR 2019;3:130-133
Cherry Garg ${ }^{1}$ Suryaveer Singh ${ }^{1}$

Address for correspondence Saurabh Gupta, MD, Assistant Professor, Department of Radiology, Dayanand Medical College and Hospital, Ludhiana, Punjab141001, India (e-mail: drsaurabhjmu@gmail.com).

\author{
Abstract \\ Keywords \\ - bronchial artery \\ embolization \\ - anterior spinal artery \\ infarct \\ - monoparesis
}

Bronchial artery embolization (BAE) is an established minimally invasive treatment for massive or recurrent haemoptysis. Anterior spinal artery (ASA) infarct is an uncommon but serious complication of the procedure. The interventional radiologist doing these procedures must be well versed with the bronchial artery anatomy, its variations, and its relation with ASA to avoid this dreaded complication. The authors report a case in which the patient developed monoparesis and mild transient autonomic dysfunction post BAE but magnetic resonance imaging of spine showed diffuse symmetric cord involvement in ASA territory from D1-D4 level.

\section{Introduction}

Bronchial artery embolization (BAE) is an established minimally invasive treatment for massive or recurrent hemoptysis. Anterior spinal artery (ASA) infarct is an uncommon but serious complication of the procedure. The interventional radiologist doing these procedures must be well versed with the bronchial artery anatomy, its variations, and its relation with ASA to avoid this dreaded complication. We report a case in which the patient developed monoparesis and mild transient autonomic dysfunction post BAE but magnetic resonance imaging (MRI) of spine showed diffuse symmetric cord involvement in ASA territory from D1-D4 level.

\section{Case Report}

A 62-year-old diabetic hypertensive male patient with history of treated pulmonary Koch's infection presented with cough and recurrent mild hemoptysis. Patient was admitted and was managed conservatively. Chest X-ray revealed patchy areas of fibrobronchiectasis in both lobes (-Fig. 1). An evening before planned discharge, the patient had an episode of massive hemoptysis following which he was taken up for BAE after informed consent. Preprocedure computed tomography (CT) angiography was not done in view of X-ray changes and due to financial constraints of the family.

received

March 5, 2019

accepted after revision

April 29, 2019

published online

July 26, 2019
Through femoral vascular access the right intercostobronchial trunk (ICBT) was catheterized with a 5F RC-1 (Cook Medical Inc.) catheter and angiography was performed which revealed abnormal parenchymal blush with bronchopulmonary shunting in right upper lobe ( - Fig. 2a, b). Similar abnormal vascularity was seen in left upper lobe through

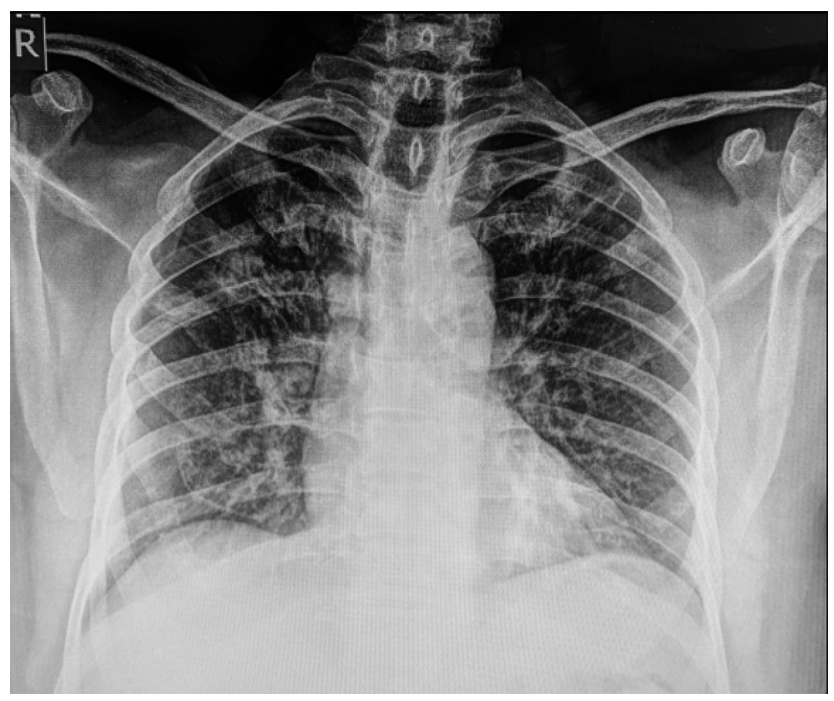

Fig. 1 X-ray chest posteroanterior view showing patchy fibrobronchiectatic changes in both upper and middle zones, predominant on right side. 


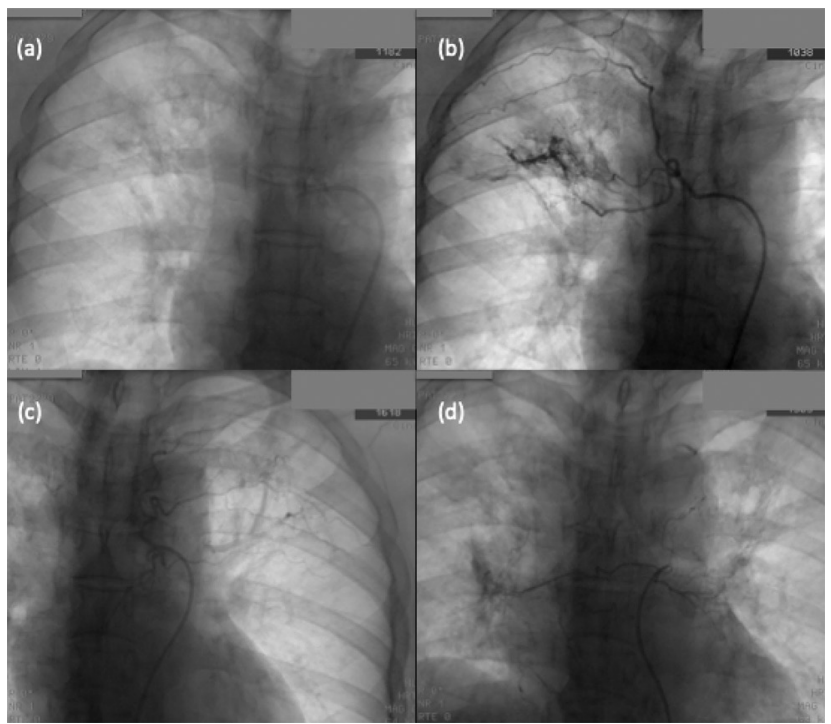

Fig. 2 (a) Right upper lobe fibrobronchiectatic changes with angiographic catheter at ostium of right ICBT. (b) Angiogram from right ICBT showing abnormal parenchymal blush with bronchopulmonary shunting. (c) Angiogram from left ICBT showing mild parenchymal blush with bronchopulmonary shunting. (d) Angiogram from common origin of both bronchial arteries showing abnormal parenchymal blush, mainly on right side. left ICBT ( - Fig. 2c) and mild abnormal parenchymal blush through both bronchial arteries having a common origin (-Fig. 2d). After thorough evaluation of the angiograms to rule out opacification of ASA, embolization was performed using 500-700 $\mu \mathrm{m}$ and 350-500 $\mu \mathrm{m}$ polyvinyl alcohol (PVA) particles (Boston Scientific) till the parenchymal blush was gone. No ASA feeder opacified in intermittent check angiograms. Sheath was removed and right leg was immobilized with a compression dressing over the puncture site. Post procedure, the patient complained of dull aching pain in the left lower limb. However, sensory and motor functions in left limb were normal.

The following day the patient complained of weakness in the right lower limb and was immediately referred back to rule out any local neural injury. Sonographic examination of the puncture and right lower limb Doppler were unremarkable. Nerve conduction study was normal. Patient also developed bladder dysfunction and neurological examination revealed upgoing plantar, loss of deep tendon reflexes, power $2 / 5$ on right side, and relatively cold right foot. MRI spine revealed T2 hyper intensity suggesting infarct in the ASA territory, extending from D1-D4 level ( - Fig. 3).

Patient was started on steroids; bladder function improved and right lower limb power improved to Grade 3/5. He was

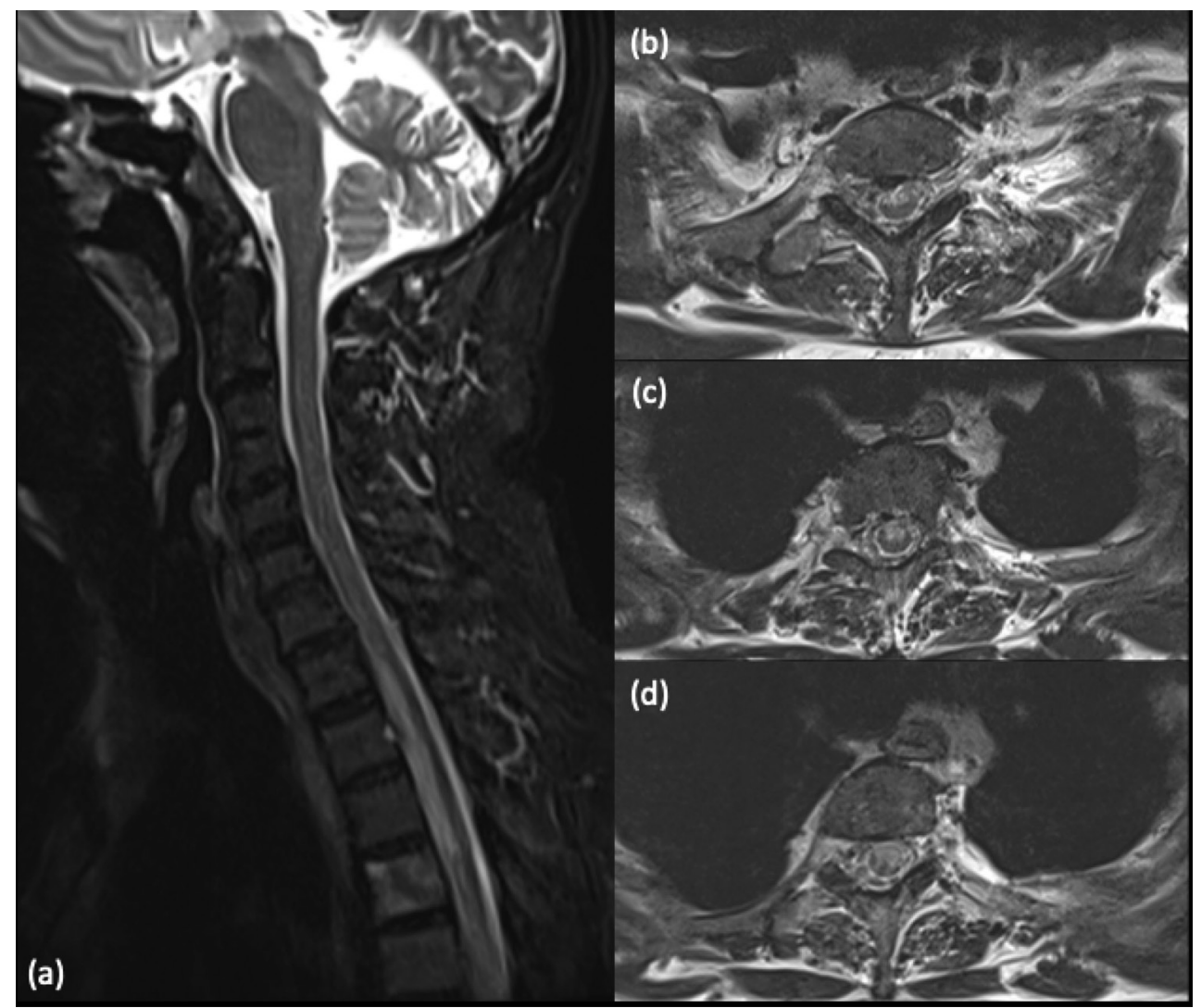

Fig. 3 (a) Short Tau Inversion Recovery (STIR) sagittal magnetic resonance imaging (MRI) section of cervicodorsal spine showing hyperintensity in anterior two-thirds of the dorsal cord extending from D1-D4 level. (b-d) T2 axial MRI sections at various levels from D1-D4 showing bilaterally symmetric hyperintensity in anterior two-thirds, consistent with anterior spinal artery territory infarct. 
discharged on tapering dose of oral steroids and was advised follow-up after a week. No recurrent episode of hemoptysis was noted till discharge. It was also decided to repeat MRI dorsal spine after review; however, the patient never reported back.

\section{Discussion}

Bronchial artery embolization is widely accepted minimally invasive procedure for patients expectorating massive amounts of blood.

\section{Relevant Vascular Anatomy}

Bronchial arteries that arise between superior endplate of D5 vertebral body and inferior endplate of D6 vertebral body are termed as orthotopic, and angiographically they are within $1 \mathrm{~cm}$ above or below the left main bronchus where it crosses descending aorta. Arteries arising from other locations are termed as ectopic (8.3-56\%), the usual sites being the inferior aortic arch proximal to ligamentum arteriosum, distal descending thoracic aorta, inferior phrenic artery, subclavian artery, brachiocephalic trunk, thyrocervical trunk, internal mammary artery, and coronary artery. ${ }^{1}$

Anterior spinal artery courses longitudinally along the anterior sulcus of the spinal cord from foramen magnum to conus medularis and supplies anterior two-thirds of cord. It is formed by confluence of descending branches of the intracranial segments of the vertebral arteries and is joined by multiple feeders from the radiculomedullary branches of the spinal trunks of segmental arteries in cervicothoracic, midthoracic and thoracolumbar regions. Midthoracic and thoracolumbar feeders to the ASA are variable and unpredictable. Angiographically small radicular arteries in upper and midthoracic region are difficult to visualize.,3 The artery of Adamkiewicz (AKA) is the most important anterior radiculomedullary feeder to the ASA and angiographically has a classic "hairpin" loop when it reaches the ASA. AKA is about 0.5 to $1.0 \mathrm{~mm}$ in diameter and almost always arises on the left side between T8-L2 (75\%).

Angiographic demonstration of nonopacification of the radiculomedullary feeders to ASA from common ICBT is very important during BAE as thoracic and upper lumbar portions of spinal cord have minimal collateral circulation, thus vulnerable to any ischemic compromise. ${ }^{2,3}$

\section{Bronchial Artery Embolization}

Bronchial artery embolization is performed through bronchial artery or ICBT demonstrating angiographic abnormal parenchymal blush and various projections are taken to rule out opacification of ASA or AKA. Agents used for embolization are gelfoam, PVA, and n-butyl-2-cyanoacrylate (NBCA) to achieve good distal embolization. Theoretically, using larger PVA particles ( $>350 \mu \mathrm{m}$ ) is a safeguard for avoiding nontargeted spinal artery embolization. ${ }^{4}$ Coils are not preferred mode of embolization as putting coil would "close the door" for any future intervention. ${ }^{5}$ Some authors have also shown better 1-year, 3-year, and 5-year hemoptysis-free periods with NBCA. ${ }^{6}$ Use of microcatheter is recommended to avoid central reflux and nontargeted embolization especially if injecting embolizing agent from common ICBT; however, a study has shown that small invisible radiculomedullay feeders to the ASA may still get embolized even with use of microcatheter. ${ }^{7}$

\section{Anterior Spinal Artery Infarct}

Anterior spinal artery infarct is a rare but dreaded complication associated with BAE. Incidence is reported between 1.0 and $6.5 \%$ and patients usually present with ASA syndrome. ${ }^{8}$ Patients have bilateral lower limb motor loss, loss of pain, and temperature sensations below the level of the lesion with relative sparing of proprioception and vibratory sensations. Autonomic dysfunctions like hypotension, sexual dysfunction, and bladder-bowel dysfunction may also be present. Deep tendon reflexes are absent in acute phase; however in few days/weeks spasticity and hyper-reflexia ensue.

Spinal artery infraction post BAE has also been reported even in patients in whom spinal artery feeders were not identified on angiography and this has been implicated to redirection of the blood to invisible connections once hypertrophied vessels start getting embolized. ${ }^{9}$

Monoparesis with mild autonomic dysfunction with symmetric ASA territory cord changes in MRI has not been described in English literature. Few authors have described transient lower limb monoparesis but they implicated it to particle reflux in main aorta. ${ }^{10} \mathrm{Few}$ sporadic reports on monoparesis due to spinal infarct post abdominal surgical procedures have been described; however, the cord involvement in them had been focal and unilateral. ${ }^{11,12}$ Some authors have also attributed unilateral symptoms in ASA infarcts in their studies to duplication of anterior spinal artery and in those patients MRI T2 hyperintensity was localized to one side. ${ }^{13,14}$ This is possibly the first case report with diffuse symmetric spinal cord changes in territory of anterior spinal artery and patient presenting with unilateral weakness. Explanation to the presentation could be acute edematous T2 hyperintensity in the region surrounding the ischemic insult and sparing of the motor tracts, probably due to duplication of the ASA in our patient which was not evaluated by us on imaging; however, it is reported in literature., ${ }^{2,13}$

\section{Conflict of Interest}

None declared.

\section{References}

1 Walker CM, Rosado-de-Christenson ML, Martínez-Jiménez S, Kunin JR, Wible BC. Bronchial arteries: anatomy, function, hypertrophy, and anomalies. Radiographics 2015;35(1):32-49

2 Miyasaka K, Asano T, Ushikoshi S, Hida K, Koyanagi I. Vascular anatomy of the spinal cord and classification of spinal arteriovenous malformations. Interv Neuroradiol 2000;6(Suppl 1): 195-198

3 Santillan A, Nacarino V, Greenberg E, et al. Vascular anatomy of the spinal cord. J Neurointervent Surg 2012; 4(1):67-74

4 Mauro MA, Jaques PF, Morris S. Bronchial artery embolization for control of hemoptysis. Semin Intervent Radiol 1992;9:45-51

5 Hartnell GG. Embolization in the treatment of acquired and congenital abnormalities of the heart and thorax. Radiographics 1993;13(6):1349-1362 
6 Woo S, Yoon CJ, Chung JW, et al. Bronchial artery embolization to control hemoptysis: comparison of N-butyl-2cyanoacrylate and polyvinyl alcohol particles. Radiology 2013;269(2):594-602

7 Brown AC, Ray CE. Anterior spinal cord infarction following bronchial artery embolization. Semin Intervent Radiol 2012;29(3):241-244

8 Yoon W, Kim JK, Kim YH, Chung TW, Kang HK. Bronchial and nonbronchial systemic artery embolization for life-threatening hemoptysis: a comprehensive review. Radiographics 2002;22(6):1395-1409

9 Wong ML, Szkup P, Hopley MJ. Percutaneous embolotherapy for life-threatening hemoptysis. Chest 2002;121(1):95-102

10 Agmy GM, Wafy SM, Mohamed SAA, et al. Bronchial and nonbronchial systemic artery embolization in management of hemoptysis: experience with 348 patients. Int Sch Res Not. 2013;26:e263259. Doi: 10.1155/2013/263259

11 Tanaka H, Minatoya K, Matsuda H, et al. Embolism is emerging as a major cause of spinal cord injury after descending and thoracoabdominal aortic repair with a contemporary approach: magnetic resonance findings of spinal cord injury. Interact Cardiovasc Thorac Surg 2014;19(2):205-210

12 Helsen G, Das V, Dobbeleir I, Van Sonhoven F. Pure motor unilateral leg paresis caused by a spinal cord infarction Acta Neurol Belg 2017;117(2):559 560

13 Romanes GJ. The arterial blood supply of the human spinal cord. Paraplegia 1965;2:199-207

14 Gwak DS, Kim HR, Jeong HY, et al. Cervical spinal cord infarction presenting as unilateral weakness. J Neurocrit Care 2014;7:60-62 